PROCEEDINGS OF THE

AMERICAN MATHEMATICAL SOCIETY

Volume 127, Number 2, February 1999, Pages 409-415

S 0002-9939(99)04452-4

\title{
THE DEGREE OF COCONVEX POLYNOMIAL APPROXIMATION
}

\author{
K. KOPOTUN, D. LEVIATAN, AND I. A. SHEVCHUK
}

(Communicated by J. Marshall Ash)

\begin{abstract}
Let $f \in C[-1,1]$ change its convexity finitely many times in the interval, say $s$ times, at $Y_{s}:-1<y_{1}<\cdots<y_{s}<1$. We estimate the degree of approximation of $f$ by polynomials of degree $n$, which change convexity exactly at the points $Y_{s}$. We show that provided $n$ is sufficiently large, depending on the location of the points $Y_{s}$, the rate of approximation is estimated by the third Ditzian-Totik modulus of smoothness of $f$ multiplied by a constant $C(s)$, which depends only on $s$.
\end{abstract}

\section{INTRODUCTION AND MAIN RESULTS}

Let $f \in C[-1,1]$ change its convexity finitely many times, say $s$, at the points $Y_{s}$ : $-1<y_{1}<\cdots<y_{s}<1$ in $[-1,1]$. For later reference set $y_{0}:=-1$ and $y_{s+1}:=1$. Note that if $f \in C^{2}[-1,1]$, then the above is equivalent to $f^{\prime \prime}(x) \prod_{i=1}^{s}\left(x-y_{i}\right) \geq 0$, in $[-1,1]$. We wish to approximate $f$ by means of polynomials which are coconvex with $f$, that is, which change convexity exactly at the points $Y_{s}$.

Questions of this nature first appeared in the work of D. J. Newman and his students (see $[\mathrm{NPR}],[\mathrm{PR}],[\mathrm{PRR}]$ ). They dealt not with a function $f$, changing convexity, but rather with $f$ that changes monotonicity finitely many times in $[-1,1]$ and they were able to obtain weaker Jackson type estimates on the degree of approximation of that function by polynomials which were truly comonotone with it as well as some proper Jackson estimates when the polynomials were comonotone with $f$ except near the points where a change of monotonicity of $f$ occurred. Later Newman $[\mathrm{N}]$ obtained the proper Jackson estimate involving the modulus of continuity of $f$ for the approximation by polynomials that were truly comonotone with $f$ (see also Iliev [I] for some relevant work). Then Leviatan and Beatson [BL] obtained the desired estimates under the assumption that $f$ possesses a continuous derivative in $[-1,1]$, applying a flipping technique and induction assumption on the number of monotonicity changes of $f$. We will apply their technique in this paper.

Received by the editors May 9, 1996 and, in revised form, April 1, 1997.

1991 Mathematics Subject Classification. Primary 41A10, 41A17, 41A25, 41A29.

Key words and phrases. Coconvex polynomial approximation, Jackson estimates.

The first author acknowledges partial support by the Izaak Walton Killam Memorial Scholarship.

The second author acknowledges partial support by ONR grant N00014-91-1076 and by DoD grant N00014-94-1-1163.

The third author was partially supported by the State Fund for Fundamental Research of Ukraine.

(C)1999 American Mathematical Society 
The first Jackson estimate involving the Ditzian-Totik moduli of smoothness (defined below) is due to Leviatan $[\mathrm{L}]$ whose estimates were later improved by Kopotun $[\mathrm{K}]$. As was pointed out to us by the referee, the degree of coconvex polynomial approximation apparently was first discussed in the Ph.D. dissertation of Diane C. Myers $[\mathrm{M}]$ where she obtained the Jackson estimate $\omega(f, 1 / n)$ for "nearly" coconvex approximation. For proper coconvex approximation there have been no results except for a short remark in [BL] that the technique employed there would give an estimate for the approximation of twice continuously differentiable functions with a single change of convexity.

The first estimates on the degree of coconvex approximation for a twice continuously differentiable function with an arbitrary finite number of convexity changes have recently been obtained by Kopotun [K1] and the purpose of this note is to improve those results in that we do not assume the existence everywhere, and continuity of even the first derivative. We are going to make use of some special polynomials related to the function $f$ which were constructed in that article [K1] based upon the polynomials introduced by Shevchuk [S]. We remark that in the above mentioned paper, Kopotun was able to obtain estimates on the simultaneous approximation of the function and its derivatives by the polynomials and their derivatives, thus obtaining, simultaneously, coconvex approximation to $f$ and comonotone approximation to its derivative. In order to state our main result we recall the definition of the $m$ th order Ditzian-Totik moduli of smoothness $\omega_{m}^{\varphi}(f, t)$. For $f \in C[-1,1]$, we set

$$
\omega_{m}^{\varphi}(f, t)=\sup _{0<h \leq t}\left\|\Delta_{h \varphi(\cdot)}^{m} f(\cdot)\right\|,
$$

where the norm is the max-norm, $\varphi(x):=\sqrt{1-x^{2}}$, and

$$
\Delta_{\eta}^{m} f(x):= \begin{cases}\sum_{i=0}^{m}\left(\begin{array}{c}
m \\
i
\end{array}\right)(-1)^{m-i} f\left(x-\frac{m}{2} \eta+i \eta\right), & \text { if } x \pm \frac{m}{2} \eta \in[-1,1], \\
0, & \text { otherwise. }\end{cases}
$$

is the symmetric $m$ th difference.

Our main result is the following.

Theorem. Let $f \in C[-1,1]$ have $s$ changes of convexity at $Y_{s}:-1<y_{1}<\cdots<$ $y_{s}<1$, and denote $d\left(Y_{s}\right):=\min \left\{1+y_{1}, y_{2}-y_{1}, \ldots, y_{s}-y_{s-1}, 1-y_{s}\right\}$. Then there exists a constant $A=A(s)$ which depends only on the number of convexity changes $-s$, such that, for $n>\frac{A(s)}{d\left(Y_{s}\right)}$, there is a polynomial $p_{n}$ of degree not exceeding $n$, which is coconvex with $f$ and satisfies

$$
\left\|f-p_{n}\right\| \leq C(s) \omega_{3}^{\varphi}\left(f, n^{-1}\right),
$$

whence,

$$
\left\|f-p_{n}\right\| \leq C(s) \omega_{3}(f, 1 / n) .
$$

The constant $C(s)$ is independent of $f$ and $n$ and of the location of the convexity changes.

Remark. Estimate (1) is best possible in that one cannot replace $\omega_{3}^{\varphi}\left(f, n^{-1}\right)$ by any higher modulus of smoothness, even not with the larger ordinary modulus of smoothness. This is due to Shvedov [Sv] in case $f$ is convex and to Zhou [Z] in case $f$ has convexity changes.

While it is obvious that the constants $C(s)$ and $A(s)$ depend on $s$, we have no estimate as to how fast they grow with it. For instance, one readily observes that 
the proof below yields exponential growth of $C(s)$ as a function of $s$ (due to the induction process), but we do not know that this is actually the case.

Even the following immediate consequences of the theorem are new:

Corollary. Let $f$ be as in the theorem and let $n>\frac{A(s)}{d\left(Y_{s}\right)}$. If in addition $f^{(r-1)} \in$ $A C[-1,1]$, and $f^{(r)} \in L_{\infty}, r=1,2,3$, then the same polynomial $p_{n}$ satisfies

$$
\left\|f-p_{n}\right\| \leq \frac{C(s)}{n^{r}}\left\|f^{(r)}\right\|_{\infty},
$$

where the norm on the right is the $L_{\infty}$-norm. (The case $r=3$ follows from [K1].)

In the sequel we will denote by $C$ an absolute constant which may vary from one occurrence to another even in the same line. Similarly $C(\cdot)$ will denote a constant which depends on a specific parameter but may change from one occurrence to another.

\section{Proof of the theorem}

If $f$ has no change of convexity in $[-1,1]$, i.e., $s=0$ and $f$ is convex in $[-1,1]$, then the theorem is valid and it was proved by Kopotun $[\mathrm{K}]$; thus we will assume that $s \geq 1$. We first need to construct a smoother function at $y_{1}$. The function $f$ is either concave or convex in $\left[-1, y_{1}\right]$, and each case will need a separate though similar construction. We will detail the construction for the case where $f$ is concave in $\left[-1, y_{1}\right]$. For the sake of simplicity in notation in the sequel we write $\alpha:=y_{1}$. Now let $x_{j}:=x_{j, n}:=\cos \frac{j \pi}{n}, j=0, \ldots, n$, be the Chebyshev nodes; and denote $I_{j}:=\left[x_{j}, x_{j-1}\right], h_{j}:=h_{j, n}:=x_{j-1}-x_{j}$ and

$$
\psi_{j}(x):=\psi_{j, n}(x):=\frac{h_{j}}{\left|x-x_{j}\right|+h_{j}} .
$$

It is well known that $h_{j \pm 1}<3 h_{j}$ and that for $x \in I_{j} \Delta_{n}(x) \leq h_{j}<5 \Delta_{n}(x)$, where, as always, $\Delta_{n}(x):=\frac{\sqrt{1-x^{2}}}{n}+\frac{1}{n^{2}}$. We assume that $\alpha \in\left[x_{j_{0}}, x_{j_{0}-1}\right)$. Then, if $n>N_{\alpha}:=\max \left\{\frac{50}{y_{2}-\alpha}, \frac{50}{1+\alpha}\right\}$, we are assured that $x_{j_{0}+3} \geq-1$ and that $x_{j_{0}-4} \leq y_{2}$. Set $h:=c \Delta_{n}(\alpha)<\frac{1}{6} h_{j_{0}}$, where $c$ is chosen sufficiently small to guarantee the right inequality. Note that this implies $x_{j_{0}+1}<\alpha-2 h<\alpha+2 h<x_{j_{0}-2}$.

We are going to replace $f$ on the interval $[\alpha-h, \alpha+h]$ in a way that will keep us near the original function and at the same time the new function (see $\hat{g}$ below) will be smoother at $\alpha$. As was mentioned above, we will apply a flipping technique and induction on the number of convexity changes of $f$. The case $s=0$ is known and serves as the beginning of the induction process; it has been proved by Kopotun $[\mathrm{K}]$. When $s=0$, then (1) holds for all $n \geq 2$. Thus, we proceed by induction.

To this end we note that either $\Delta_{h}^{2} f(\alpha) \geq 0$, or $\Delta_{h}^{2} f(\alpha)<0$.

In the first case, let $L_{1}(x)$ denote the linear function interpolating $f$ at $\alpha-h$ and $\alpha$. Then the function $g:=f-L_{1}$ satisfies

$$
g(\alpha-h)=g(\alpha)=0, \quad g(\alpha+h) \geq 0, \quad \text { and } g(x) \leq 0, \quad-1 \leq x<\alpha-h .
$$

Hence, for $J_{0}:=\left[x_{j_{0}+1}, x_{j_{0}-2}\right]$, we have,

$$
\begin{aligned}
0 \leq g(\alpha+h) & \leq g(\alpha+h)-g(\alpha-2 h) \\
& =g(\alpha+h)-3 g(\alpha)+3 g(\alpha+h)-g(\alpha-2 h) \\
& \leq \omega_{3}\left(g, h ; J_{0}\right)=\omega_{3}\left(f, h ; J_{0}\right) .
\end{aligned}
$$


Similarly, in the latter case, let $L_{1}(x)$ denote the linear function interpolating $f$ at $\alpha$ and $\alpha+h$. Then the function $g:=f-L_{1}$ satisfies

$$
g(\alpha+h)=g(\alpha)=0, \quad g(\alpha-h)<0, \quad \text { and } \quad g(x) \geq 0, \quad \alpha+h \leq x<y_{2} .
$$

Hence,

$$
\begin{aligned}
0<-g(\alpha-h) & \leq g(\alpha+2 h)-g(\alpha-h) \\
& =g(\alpha+2 h)-3 g(\alpha+h)+3 g(\alpha)-g(\alpha-h) \\
& \leq \omega_{3}\left(g, h ; J_{0}\right)=\omega_{3}\left(f, h ; J_{0}\right) .
\end{aligned}
$$

Thus, in both cases we have,

$$
\max \{|g(\alpha-h)|,|g(\alpha)|,|g(\alpha+h)|\} \leq C \omega_{3}\left(f, h ; J_{0}\right),
$$

which in turn implies that the quadratic polynomial $L_{2}(x)$, interpolating $g$ at $\alpha-h$, $\alpha$ and $\alpha+h$, is bounded by the same on $[\alpha-h, \alpha+h]$. Thus

$$
\left|L_{2}(x)\right| \leq C\left(\frac{|x-\alpha|+h_{j_{0}}}{h_{j_{0}}}\right)^{2} \omega_{3}\left(f, h ; J_{0}\right), \quad x \in[-1,1] .
$$

At the same time applying Whitney's Theorem we conclude that

$$
\left|g(x)-L_{2}(x)\right| \leq C \omega_{3}\left(f, h ; J_{0}\right), \quad x \in[\alpha-h, \alpha+h] .
$$

Now it follows by Lemma 4.2 of [S1] that

$$
\left|g(x)-L_{2}(x)\right| \leq C\left(\frac{|x-\alpha|+h_{j_{0}}}{h_{j_{0}}}\right)^{3} \omega_{3}\left(f, h ; J_{0}\right), \quad x \in[-1,1] .
$$

Observing that

$$
\omega_{3}\left(f, h ; J_{0}\right) \leq C \omega_{3}^{\varphi}\left(f, n^{-1}\right)
$$

we obtain by (3) and (4),

$$
|g(x)| \leq C \psi_{j_{0}}^{-3} \omega_{3}^{\varphi}\left(f, n^{-1}\right), \quad x \in[-1,1] .
$$

Now let

$$
\tilde{g}(x)= \begin{cases}-g(x), & x \in[-1, \alpha] \\ g(x), & \text { otherwise }\end{cases}
$$

and finally,

$$
\hat{g}(x)= \begin{cases}\tilde{g}(x), & x \notin[\alpha-h, \alpha+h], \\ \max \{\tilde{g}(x), 0\}, & x \in[\alpha-h, \alpha+h] .\end{cases}
$$

By virtue of (5) we immediately have

$$
\|\tilde{g}-\hat{g}\| \leq C \omega_{3}^{\varphi}\left(f, n^{-1}\right) .
$$

Also

$$
\begin{aligned}
\omega_{3}^{\varphi}\left(\hat{g}, n^{-1}\right) & \leq \omega_{3}^{\varphi}\left(\tilde{g}, n^{-1}\right)+C \omega_{3}^{\varphi}\left(f, n^{-1}\right) \\
& \leq C \omega_{3}^{\varphi}\left(f, n^{-1}\right),
\end{aligned}
$$

where the first inequality follows from (6), while the second is due to the fact that for $x \notin J_{0}$,

$$
\left|\Delta_{\varphi(x) / n}^{2} \tilde{g}(x)\right|=\left|\Delta_{\varphi(x) / n}^{2} g(x)\right|=\left|\Delta_{\varphi(x) / n}^{2} f(x)\right|,
$$

and for $x \in J_{0}$ we apply (5). 
It is readily seen that $\hat{g} \in C[-1,1]$, that it is convex in $\left[-1, y_{2}\right]$ and that it changes convexity at $Y_{s-1}^{\prime}:=Y_{s} \backslash\left\{y_{1}\right\}$. If, on the other hand, $f$ was convex in $\left[-1, y_{1}\right]$, then $\hat{g}$ would be concave in $\left[-1, y_{2}\right]$ and change convexity at $Y_{s-1}^{\prime}$. Thus in any case $\hat{g}$ has fewer convexity changes, so by induction, we may assume that for $n>\frac{A(s-1)}{d\left(Y_{s-1}^{\prime}\right)}$, there exists an $n$th degree polynomial $q_{n}$ which is coconvex with $\hat{g}$, and which satisfies the analogue of (1). Namely (by (7)),

$$
\left\|\hat{g}-q_{n}\right\| \leq C(s-1) \omega_{3}^{\varphi}\left(\hat{g}, n^{-1}\right) \leq C(s) \omega_{3}^{\varphi}\left(f, n^{-1}\right) .
$$

Thus, fixing $n>\max \left\{\frac{A(s-1)}{d\left(Y_{s-1}^{\prime}\right)}, N_{\alpha}\right\}$ readily leads to the definition of $A(s)$. Note that since $\hat{g}(\alpha)=0$, we may assume that $q_{n}(\alpha)=0$ doubling the constant in (8).

Kopotun [K1] has constructed for $\alpha$ and $q_{n}$ for each $n$ as above, two polynomials $V_{n}$ and $W_{n}$ of degrees at most $20 n(s+1)=: 4 n \mu$, with the properties that for all $x \in[-1,1]$,

$$
\begin{gathered}
V_{n}(x) \operatorname{sgn}(x-\alpha) \geq 0, \\
V_{n}^{\prime}(x) q_{n}^{\prime \prime}(x)\left(q_{n}^{\prime}(x)-q_{n}^{\prime}(\alpha)\right) \operatorname{sgn}(x-\alpha) \geq 0, \\
\left|V_{n}(x)-\operatorname{sgn}(x-\alpha)\right| \leq C(s) \psi_{j_{0}}^{\mu}, \\
\left|W_{n}(x)-\operatorname{sgn}(x-\alpha)\right| \leq C(s) \psi_{j_{0}}^{\mu} ; \\
\left|V_{n}^{\prime}(x)\right| \leq C(s) \psi_{j_{0}}^{\mu} h_{j_{0}}^{-1} ;
\end{gathered}
$$

and finally,

$$
W_{n}^{\prime}(x) \operatorname{sgn} q_{n}^{\prime}(\alpha) \leq 0, \quad x \in\left[y_{2 j}, y_{2 j+1}\right], \quad j=0, \ldots,[s / 2],
$$

and

$$
W_{n}^{\prime}(x) \operatorname{sgn} q_{n}^{\prime}(\alpha) \geq 0, \quad x \in\left[y_{2 j+1}, y_{2 j+2}\right], \quad j=0, \ldots,[(s-1) / 2] .
$$

We are ready to define the polynomial

$$
p_{n}(x):=\int_{\alpha}^{x}\left[\left(q_{n}^{\prime}(u)-q_{n}^{\prime}(\alpha)\right) V_{n}(u)+q_{n}^{\prime}(\alpha) W_{n}(u)\right] d u,
$$

of degree at most $5 n \mu$, which evidently is coconvex with $f$. Note that $P_{n}(x):=$ $L_{1}(x)+p_{n}(x)$ is of the same degree and it too is coconvex with $f$. Hence, we conclude the induction step by proving (1) for $P_{n}$.

We begin with

$$
\begin{aligned}
\left|f(x)-P_{n}(x)\right|= & \left|g(x)-p_{n}(x)\right|=\left|\tilde{g}(x) \operatorname{sgn}(x-\alpha)-p_{n}(x)\right| \\
\leq & \|\tilde{g}-\hat{g}\|+\left|\hat{g}(x) \operatorname{sgn}(x-\alpha)-p_{n}(x)\right| \\
\leq & C \omega_{3}^{\varphi}\left(f, n^{-1}\right)+\left|\left(\hat{g}(x)-q_{n}(x)\right) \operatorname{sgn}(x-\alpha)\right|+\mid q_{n}(x) \operatorname{sgn}(x-\alpha) \\
& -\int_{\alpha}^{x} q_{n}^{\prime}(u) V_{n}(u) d u|+| q_{n}^{\prime}(\alpha) \int_{\alpha}^{x}\left(V_{n}(u)-W_{n}(u)\right) d u \mid \\
= & : E_{1}+E_{2}+E_{3}+E_{4}, \text { say. }
\end{aligned}
$$

By virtue of (8),

$$
E_{2} \leq C(s) \omega_{3}^{\varphi}\left(f, n^{-1}\right) .
$$


Recalling that $q_{n}(\alpha)=0$, integration by parts and (11) yield

$$
\begin{aligned}
E_{3} & =\left|q_{n}(x)\left(\operatorname{sgn}(x-\alpha)-V_{n}(x)\right)+\int_{\alpha}^{x} q_{n}(u) V_{n}^{\prime}(u) d u\right| \\
& \leq C(s)\left(\left|q_{n}(x)\right| \psi_{j_{0}}^{\mu}+\int_{\alpha}^{x}\left|q_{n}(u)\right| h_{j_{0}}^{-1} \psi_{j_{0}}^{\mu}(u) d u\right) \\
& \leq C(s) \omega_{3}^{\varphi}\left(f, n^{-1}\right),
\end{aligned}
$$

where the last inequality in (13) follows from (applying (5), (6) and (7)),

$$
\begin{aligned}
\left|q_{n}(x)\right| & \leq|\tilde{g}(x)|+\left|\tilde{g}(x)-q_{n}(x)\right| \\
& \leq|g(x)|+\|\tilde{g}-\hat{g}\|+\left\|\hat{g}-q_{n}\right\| \\
& \leq C \psi_{j_{0}}^{-3} \omega_{3}^{\varphi}\left(f, n^{-1}\right),
\end{aligned}
$$

and the easy inequality

$$
\int_{\alpha}^{x} \psi_{j_{0}}^{\nu}(u) d u \leq C h_{j_{0}}, \quad \nu \geq 2 .
$$

Finally, in order to estimate $E_{4}$, we need an estimate on $q_{n}^{\prime}(\alpha)$. To this end we observe that since $q_{n}$ is convex in $\left[-1, y_{2}\right]$, then $q_{n}^{\prime}$ is monotone increasing there. If $q_{n}^{\prime}(\alpha) \geq 0$, then by $(5)$, for some $\xi \in\left(\alpha, \alpha+h_{j_{0}}\right)$,

$$
\begin{aligned}
& 0 \leq q_{n}^{\prime}(\alpha) \leq q_{n}^{\prime}(\xi)=\frac{q_{n}\left(\alpha+h_{j_{0}}\right)-q_{n}(\alpha)}{h_{j_{0}}} \\
& =h_{j_{0}}^{-1} q_{n}\left(\alpha+h_{j_{0}}\right) \leq C h_{j_{0}}^{-1} \omega_{3}^{\varphi}\left(f, n^{-1}\right) .
\end{aligned}
$$

And if $q_{n}^{\prime}(\alpha)<0$, then by (5), for some $\xi \in\left(\alpha-h_{j_{0}}, \alpha\right)$,

$$
\begin{aligned}
0 \leq-q_{n}^{\prime}(\alpha) \leq-q_{n}^{\prime}(\xi) & =\frac{q_{n}\left(\alpha-h_{j_{0}}\right)-q_{n}(\alpha)}{h_{j_{0}}} \\
& =h_{j_{0}}^{-1} q_{n}\left(\alpha-h_{j_{0}}\right) \leq C h_{j_{0}}^{-1} \omega_{3}^{\varphi}\left(f, n^{-1}\right) .
\end{aligned}
$$

Hence by (9), (10) and (14),

$$
\begin{aligned}
E_{4} & \leq\left|q_{n}^{\prime}(\alpha)\right| \int_{\alpha}^{x}\left|V_{n}(u)-W_{n}(u)\right| d u \\
& \leq C h_{j_{0}}\left|q_{n}^{\prime}(\alpha)\right| \leq C \omega_{3}^{\varphi}\left(f, n^{-1}\right) .
\end{aligned}
$$

Combining (12), (13) and (15) we see that

$$
\left\|f-P_{n}\right\| \leq C \omega_{3}^{\varphi}\left(f, n^{-1}\right) .
$$

This completes our proof.

\section{REFERENCES}

[BL] R. K. Beatson and D. Leviatan, On comonotone approximation, Canadian Math. Bull. 26 (1983), 220-224. MR 84f: 41022

[I] G. L. Iliev, Exact estimates for partially monotone approximation, Analysis Math. 4 (1978), 181-197. MR 80h:41007

[K] K. A. Kopotun, Pointwise and uniform estimates for convex approximation of functions by algebraic polynomials, Constr. Approx. 10 (1994), 153-178. MR 95k:41014

[K1] K. A. Kopotun, Coconvex polynomial approximation of twice differentiable functions, J. Approx. Theory, 83 (1995), 141-156. MR 97e:41043

[L] D. Leviatan, Pointwise estimates for convex polynomial approximation, Proc. Amer. Math. Soc. 98 (1986), 471-474. MR 88i:41010 
[M] Diane Claire Myers, Comonotone and co-convex approximation, Ph.D. dissertation, Temple University: L. Raymon supervisor, 1975.

[N] D. J. Newman, Efficient comonotone approximation, J. Approx. Theory 25 (1979), 189192. MR 80j:41011

[NPR] D. J. Newman, E. Passow and L. Raymon, Piecewise monotone polynomial approximation, Trans. Amer. Math. Soc. 172 (1972), 465-472. MR 46:9604

[PR] E. Passow and L. Raymon, Monotone and comonotone approximation, Proc. Amer. Math. Soc. 42 (1974), 390-394. MR 89m:41021

[PRR] E. Passow, L. Raymon and J. A. Roulier, Comonotone polynomial approximation, J. Approx. Theory 11 (1974), 221-224. MR 50:5293

[S] I. A. Shevchuk, Approximation of monotone functions by monotone polynomials, Russ. Akad. Nauk Matem. Sbornik 183 (1992); English transl. in Russ. Acad. Sci. Sbornik Math. 76 (1993), 51-64, MR 94i:41022

[S1] I. A. Shevchuk, Approximation by polynomials and traces of functions continuous on an interval, (in Russian), Naukova Dumka, Kiev, Ukraine, 1992.

[Sv] A. S. Shvedov, Orders of coapproximation of functions by algebraic polynomials, Mat. Zametki 29 (1981) 117-130; English transl. in Math. Notes 29 (1981), 63-70. MR 82c:41009

[Z] S. P. Zhou, On comonotone approximation by polynomials in $L^{p}$ space, Analysis 13 (1993), 363-376. MR 95d:41021

Department of Mathematical Sciences, University of Alberta, Edmonton, Alberta, Canada T6G 2G1

Current address: Department of Mathematics, Vanderbilt University, Nashville, Tennessee 37240

E-mail address: kkopotun@math.vanderbilt.edu

School of Mathematical Sciences, Sackler Faculty of Exact Sciences, Tel Aviv University, Tel Aviv 69978, Israel and Department of Mathematics, University of South Carolina, Columbia, South Carolina 29208

E-mail address: leviatan@math.tau.ac.il

Institute of Mathematics, National Academy of Sciences of Ukraine, Kyiv 252601, UKRAINE

E-mail address: shevchuk@dad.imath.kiev.ua 